\title{
Upstream Ultra-Low Frequency Waves observed by MESSENGER's Magnetometer: Implications for Particle Acceleration at Mercury's Bow Shock
}

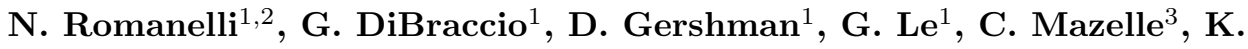 \\ Meziane $^{4}$, S. Boardsen ${ }^{1}$, J. Slavin ${ }^{5}$, J. Raines ${ }^{5}$, A. Glass ${ }^{5}$, J. Espley ${ }^{1}$ \\ ${ }^{1}$ NASA Goddard Space Flight Center, Greenbelt, Maryland, USA. \\ ${ }^{2}$ CRESST II, University of Maryland, Baltimore County, USA. \\ ${ }^{3}$ Institut de Recherche en Astrophysique et Planetologie, UPS/CNRS, Toulouse, France. \\ ${ }^{4}$ Department of Physics, University of New Brunswick, Fredericton, New Brunswick, Canada. \\ ${ }^{5}$ Department of Climate and Space Sciences and Engineering, University of Michigan, Ann Arbor, \\ Michigan, USA.
}

Key Points:

- We perform the first statistical analysis (4536 events) of the main properties of the lowest frequency waves in the Hermean foreshock.

- Small normalized wave amplitude $(\sim 0.2)$ and occurrence $(\sim 0.5 \%)$ are likely due to low backstreaming proton flux and variable external conditions.

- The normalized backstreaming protons speed $(\sim 0.95-2.6)$ suggests that similar acceleration processes occur at several planetary shocks.

This is the author manuscript accepted for publication and has undergone full peer review but has not been through the copyediting, typesetting, pagination and proofreading process, which may lead to differences between this version and the Version of Record. Please cite this article as doi: 10.1029/2020GL087350 


\section{Abstract}

We perform the first statistical analysis of the main properties of waves observed in the $0.05-0.41 \mathrm{~Hz}$ frequency range in the Hermean foreshock by the MErcury Surface, Space ENvironment, GEochemistry, and Ranging (MESSENGER) Magnetometer. Although we find similar polarization properties to the '30 second' waves observed at the Earth's foreshock, the normalized wave amplitude $\left(\delta B /\left|\mathbf{B}_{\mathbf{0}}\right| \sim 0.2\right)$ and occurrence rate $(\sim 0.5 \%)$ are much smaller. This could be associated with relatively lower backstreaming proton fluxes, the smaller foreshock size and/or less stable solar wind (SW) conditions around Mercury. Furthermore, we estimate that the speed of resonant backstreaming protons in the SW reference frame (likely source for these waves) ranges between 0.95 and 2.6 times the SW speed. The closeness between this range and what is observed at other planetary foreshocks suggests that similar acceleration processes are responsible for this energetic population and might be present in the shocks of exoplanets.

\section{Introduction}

The foreshock is the spatial region upstream of, but magnetically connected to the bow shock. Due to this connection, particles from the incoming solar wind (SW) coexist with a second population of backstreaming ions, produced by reflection of SW particles at the bow shock or leakage of plasma from downstream of the shock (e.g., Burgess et al., 2012; Eastwood et al., 2005). As they move upstream along the interplanetary magnetic field (IMF), the backstreaming particles provide a source of free energy for various plasma instabilities (e.g., Brinca, 1991; Gary, Akimoto \& Winske, 1989; Mazelle et al., 2003).

Ion reflection is a general property of high Mach number collisionless shocks (Biskamp, 1973; Burgess et al., 2012; Kennel et al., 1985; Paschmann et al., 1980; Sonnerup, 1969). The analysis of the Hermean foreshock is extremely important to investigate ion reflection and related physical processes occurring under low SW Mach numbers (e.g., Gershman et al., 2013; Masters et al., 2013; Russell et al., 1982; Slavin and Holzer, 1981). In particular, the SW Alfvénic Mach number range observed at Mercury $(\sim 4-6)$ is expected to be right at or just above the critical value, where particle reflection at the bow shock should be negligible (Kennel et al., 1985; Le et al., 2013). In the present paper we characterize properties of backstreaming ions at Mercury by studying the occurrence and 
main properties of associated ultra-low frequency (ULF) waves observed in the foreshock, based on MErcury Surface, Space ENvironment, GEochemistry, and Ranging (MESSENGER) Magnetometer (MAG) observations.

To our knowledge, there have only been a few studies focusing on the Hermean foreshock based on in-situ spacecraft observations. Fairfield and Behannon (1976) first reported Mariner 10 observations and classified Mercury upstream waves into two groups: (1) lower-frequency ( 0.1-0.2 Hz) large-amplitude waves, and (2) higher-frequency $(\sim$ $2 \mathrm{~Hz}$ ) small-amplitude waves; similar to the so-called '30 second' and '1 Hz' waves observed at the Earth's foreshock, respectively (Fairfield et al., 1974; Greenstadt et al., 1968). Le et al. (2013) performed an analysis of a survey of waves observed during an Hermean foreshock passage on 26 March 2011, and constitutes the only related study based on MESSENGER orbital data so far. In particular, the authors found that the lowest frequency waves had small amplitudes $\left(\delta B /\left|\mathbf{B}_{\mathbf{0}}\right| \sim 0.1\right)$, a frequency $\sim 0.3 \mathrm{~Hz}$, and were present sporadically in Mercury's foreshock.

Although no data was presented for Mercury except an estimate from Fairfield and Behannon (1976), Hoppe and Russell (1982) found that there is a linear relationship between the observed wave frequency (of the lowest frequency mode) and the magnetic field strength for foreshock encounters around several planets, suggesting that such wave frequencies depend on local gyrofrequencies. The present study aims to extend the current state of knowledge about the Hermean foreshock by performing the first statistical study of the lowest frequency waves observed by MESSENGER MAG during all its orbital phase. Additionally, we add data to the relationship found in Hoppe and Russell (1982), we estimate the velocity of resonant backstreaming protons, and perform comparisons with other planetary foreshocks throughout the heliosphere.

\section{MESSENGER MAG Observations: A Case Study and the Wave Se- lection Criteria}

The MESSENGER spacecraft was inserted into an $~ 12$-hr period, high eccentricity $\left(\sim 200 \times 15,000-\mathrm{km}\right.$ altitude), $82^{\circ}$ inclination orbit about Mercury on 18 March 2011 (Solomon et al., 2007). The orbital period was reduced on 16 April 2012 to $\sim 8$ hr, lowering the apoapsis altitude to $\sim 4.1 \mathrm{R}_{M}$, still providing measurements upstream from the Hermean bow shock $\left(\mathrm{R}_{M}\right.$ stands for Mercury's radii equal to $2440 \mathrm{~km}$ ). The reader is referred to Figure 2 in Slavin et al. (2019) for a plot of the trajectory of MESSENGER 
over its four-year mission. Average bow shock and magnetopause fits reported in Winslow et al. (2013) are shown for comparison: the corresponding standoff distances are $1.96 \mathrm{R}_{M}$ and $1.45 \mathrm{R}_{M}$, respectively.

In this work we have analyzed all MESSENGER MAG data upstream from the Hermean bow shock with a sampling rate of $20 \mathrm{~Hz}$ (Anderson et al., 2007). We display data in the aberrated Mercury solar magnetic (MSM) coordinates. The MSM coordinate system is centered on Mercury's offset internal dipole (Anderson et al., 2011), with the XMSM axis oriented sunward along the Sun-Mercury line and the Y-MSM axis opposite to the Mercury's orbital velocity, respectively. The Z-MSM axis completes the right-handed system. We assume an aberration of $\sim 7^{\circ}$ due to Mercury's average orbital speed through a radial SW speed of $400 \mathrm{~km} \mathrm{~s}^{-1}$ to define the aberrated MSM coordinate system.

\subsection{A foreshock wave event observed at $0.283 \mathrm{~Hz}$ on 10 September 2011}

Figure 1 shows an example of the lowest frequency waves observed by MESSENGER MAG in the Hermean foreshock. These measurements were obtained on 10 September 2011, between 03:27:18.99 and 03:30:43.79 UT. MESSENGER's mean location is [0.27, $3.82,-5.81] R_{M}$. The mean magnetic field vector is $\mathbf{B}_{\mathbf{0}}=[-37.81,5.98,3.06] \mathrm{nT}$ and makes an angle of $10.06^{\circ}$ with the X-MSM axis. All magnetic field components display oscillations with a well-defined frequency. The Y-MSM and Z-MSM magnetic field components have an amplitude around $3.9 \mathrm{nT}$, the X-MSM component has an amplitude around $1.3 \mathrm{nT}$. Panel e) shows the power spectral density (PSD) for the transverse $\left(\mathbf{B}_{\perp}\right)$ and compressive $\left(\mathrm{B}_{\text {comp }}\right)$ magnetic field components with respect to $\mathbf{B}_{\mathbf{0}}$. The $\operatorname{PSD}\left(\mathbf{B}_{\perp}\right)$ displays a peak at a frequency that in the spacecraft reference frame $\left(f_{s c}\right)$ is approximately $0.283 \mathrm{~Hz}$ (vertical red dashed line). We also find that these waves are restricted mainly to the perpendicular plane to $\mathbf{B}_{\mathbf{0}}$, since $P S D\left(\mathbf{B}_{\perp}\right)>>P S D\left(B_{\text {comp }}\right)$ around $f_{s c} \sim 0.283$ Hz.

The polarization and wave vector of these low frequency waves are obtained from Minimum Variance Analysis (MVA). This technique provides an estimate of the direction of propagation for an assumed planar wave by calculating the eigenvalues of the covariance matrix of the magnetic field within a given time interval. The maximum, intermediate and minimum eigenvalues are denoted as $\lambda_{1}, \lambda_{2}$, and $\lambda_{3}$, respectively. The hypothesis that the waves are planar can be characterized by means of the $\lambda_{2} / \lambda_{3}$ ratio, 
MESSENGER MAG observations, September 10 2011, 03:27:18.99 - 03:30:43.79 UT
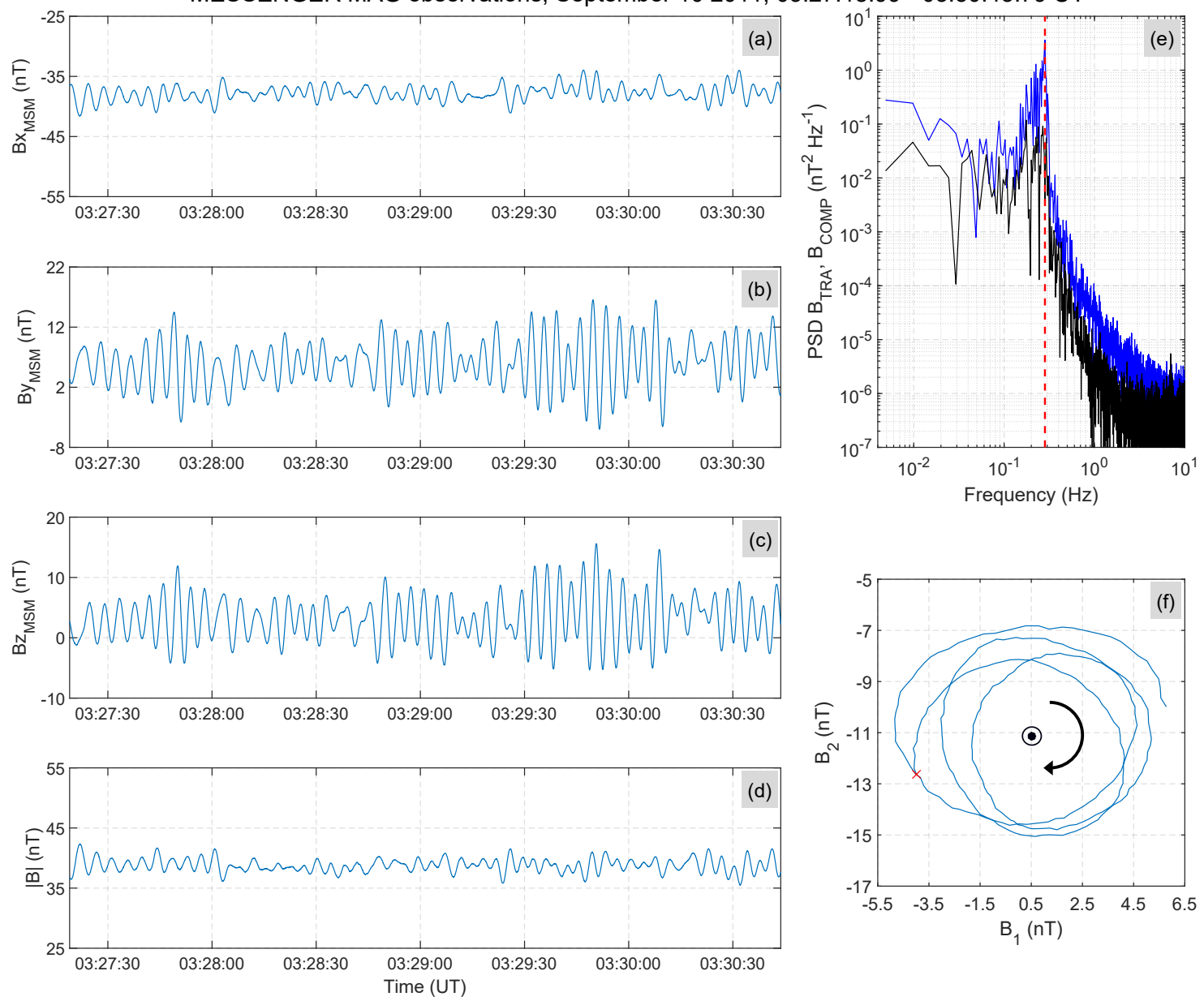

Figure 1. MESSENGER Magnetometer observations in the Hermean foreshock. Panels a) to c) display the magnetic field aberrated MSM components, panel d) shows the magnetic field intensity. Panels e) displays the power spectral density of the transverse (in blue) and compressive (in black) magnetic field components with respect to the mean magnetic field. Panel f) displays magnetic field data in the maximum-intermediate plane between 03:27:27.94 and 03:27:42.74 UT.

The red cross corresponds to the first measurement in this time interval. 
and the wave vector $\mathbf{k}$ is associated with the minimum variance eigenvector $\left(\mathbf{e}_{3}\right)$. Note that $\mathbf{e}_{3}$ defines the direction of $\mathbf{k}$ but not the sense (Sonnerup and Scheible, 1998).

Figure 1, panel f) shows the magnetic field components in the maximum-intermediate plane (hodogram), obtained by applying MVA on MAG data between 03:27:27.94 and 03:27:42.74 UT (approximately 4 wave periods). The corresponding mean magnetic field in the MVA basis $\left(\mathbf{e}_{1}, \mathbf{e}_{2}, \mathbf{e}_{3}\right)$ is $\mathbf{B}_{\mathbf{0}}=[0.56,-10.96,36.99] \mathrm{nT}$, pointing out of the maximumintermediate plane. The sense of gyration of the magnetic field oscillations (black arrow) with respect to $\mathbf{B}_{\mathbf{0}}$ indicates that the wave polarization, in the spacecraft frame is left handed. These waves are close to be circularly polarized $\left(\lambda_{1} / \lambda_{2}=1.34\right)$ and planar $\left(\lambda_{2} / \lambda_{3}=144.10\right)$. The angle $\theta_{k B}$ between the estimated wave propagation direction and $\mathbf{B}_{\mathbf{0}}$ is $16.52^{\circ}$, indicating that these waves are propagating quasi-parallel to the mean magnetic field. Moreover, by assuming that $\mathbf{k}$ points upstream we find that the angle between $\mathbf{k}$ and the $\mathrm{SW}$ velocity $\left(\theta_{k V}\right)$ is $157.96^{\circ}$. The normalized wave amplitude $\left(\delta B /\left|\mathbf{B}_{\mathbf{0}}\right|\right)$ derived based on the MVA eigenvalues (Song and Russell, 1999) is 0.08. The wave properties shown and derived from Figure 1 are all consistent with the ones reported for the case study analyzed in Le et al. (2013).

\subsection{Wave Selection Criteria}

The methodology for the statistical analysis of these waves is the following: first, we identify time intervals of $204.8 \mathrm{~s}$ with MAG observations when MESSENGER is upstream from the Hermean bow shock. These intervals, at least $\sim 10$ wave periods long, consist of 4096 measurements allowing computation of the $\operatorname{PSD}\left(\mathbf{B}_{\perp}\right)$ and $\operatorname{PSD}\left(\mathrm{B}_{\text {comp }}\right)$ based on a Fast Fourier Transform algorithm with a frequency resolution $\Delta f$ equal to $0.00488 \mathrm{~Hz}$. Overlapping between contiguous time intervals is $87.5 \%$. In addition, for each of these $204.8 \mathrm{~s}$ time intervals, we apply the MVA on MAG data over each sub-interval of $\sim 4$ observed wave periods contained in it. A wave train is often identified based on a minimum of three observed wave periods. Our criteria is slightly more strict but does not affect significantly the presented statistical results. Based on the eigenvalues and eigenvectors and derived wave polarization properties for each sub-interval, we provide the associated mean values and standard deviations for each 204.8 s time interval. A similar methodology has been considered to analyze ULF waves in the upstream region of Mars (Romanelli et al., 2016). 
We also determine whether the spacecraft was connected to the bow shock by utilizing the solar foreshock coordinates introduced by Greenstadt and Baum (1986), together with bow shock fit reported in Winslow et al. (2013). By increasing the value of the semi-latus rectum associated with the bow shock fit up to $30 \%$, we implement a conservative approach to ensure the results presented here correspond to identified events upstream from the bow shock while accounting for variability in its location. We determine MESSENGER was connected to the bow shock during each $204.8 \mathrm{~s}$ time interval, if it was continuously connected during each of the contained sub-intervals of $\sim 4$ wave periods.

We consider that a wave event of interest has been identified when MESSENGER is connected to the shock and a peak in the PSD of the MAG observations satisfies:

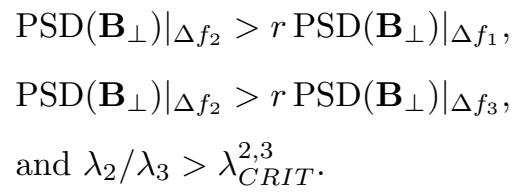
$\mathrm{Hz},[0.4199-0.5957] \mathrm{Hz}$ frequency ranges, respectively. We define $\Delta f_{2}$ as the frequency interval where the low frequency waves of interest should be observed (Fairfield and Behannon, 1976; Hoppe and Russell, 1982; Le et al., 2013). To ensure that this is the case for the majority of the wave events of interest, we restrict the analysis to cases where the mean IMF magnitude over a given $204.8 \mathrm{~s}$ time interval is equal or larger than 10 $\mathrm{nT}$. The values for $r$ and $\lambda_{C R I T}^{2,3}$ define the criteria for the detection of the lowest frequency waves, based on the wave properties. The results presented in this paper correspond to $r=4$, and $\lambda_{C R I T}^{2,3}=5$. However, we do not find significant differences when $r$ is varied between 2 and 10, and $\lambda_{C R I T}^{2,3}$ is varied between 5 and 20; and when an analogous analysis is performed considering $409.6 \mathrm{~s}$ windows $(\Delta f=0.00244 \mathrm{~Hz})$.

\section{Statistical Results and Discussion}

\subsection{Properties of Waves Observed at the $0.05-0.41 \mathrm{~Hz}$ range}

Figure 2 shows the main polarization properties of 4536 identified wave events, that is, $204.8 \mathrm{~s}$ intervals where the waves of interest are observed and fulfill the conditions specified in the previous section. Assuming that $\mathbf{k}$ points upstream, panel a) shows that these waves propagate quasi antiparallel to the $\mathrm{SW}$ velocity with $\left\langle\theta_{k V}> \pm \sigma\left(\theta_{k V}\right)=\left[164.47^{\circ} \pm\right.\right.$ 
$\left.6.50^{\circ}\right]$, where $<>$ and $\sigma$ make reference to the mean value and the standard deviation associated with the corresponding histogram, respectively. Although the direction of $\mathbf{k}$ cannot be determined with single spacecraft observations, this hypothesis is supported by the fact that the ion/ion right hand instability is most often the most unstable wave mode for tenuous field aligned beams interacting with the background plasma (e.g., Gary, 1991). Such wave mode must necessarily co-stream with the ions along the background magnetic field (i.e., points upstream) to resonate with a backstreaming proton population. This hypothesis is also in agreement with reports for the ' 30 second' waves observed at the terrestrial foreshock (e.g., Wilson et al., 2016) and hybrid simulations of the Hermean foreshock (Jarvinen et al., 2019). Panel b) shows that these waves propagate quasiparallel to the mean magnetic field direction with $\left\langle\theta_{k B}> \pm \sigma\left(\theta_{k B}\right)=\left[10.41^{\circ} \pm 4.04^{\circ}\right]\right.$. Panel c) shows that these waves are close to be circularly polarized, with $\left\langle\lambda_{1} / \lambda_{2}\right\rangle$ $\pm \sigma\left(\lambda_{1} / \lambda_{2}\right)=[1.24 \pm 0.19]$, however elliptically polarized waves are also present. Panel d) shows that they have relatively low normalized wave amplitude, with $\left\langle\delta B /\left|\mathbf{B}_{\mathbf{0}}\right|>\right.$ $\pm \sigma\left(\delta B /\left|\mathbf{B}_{\mathbf{0}}\right|\right)=(0.20 \pm 0.06)$. Moreover, we find that these waves are left handed polarized in the spacecraft reference frame.

All these wave properties are consistent with fast magnetosonic waves, intrinsically right-handed polarized in the SW reference frame, but observed with the opposite polarization due to the Doppler shift between the SW and the spacecraft rest frames. The most plausible mechanism responsible for these waves is the ion-ion right hand resonant instability, where SW backstreaming protons interact with the incoming magnetized SW plasma. Such instability satisfies approximately the cyclotron resonance condition (e.g., Brinca, 1991; Gary, Akimoto \& Winske, 1989; Mazelle et al., 2003), allowing to estimate properties of the backstreaming ions, based on the observed wave properties.

The cyclotron resonance condition between a backstreaming proton and a righthand wave is:

$$
\omega-k_{\|} V_{r}+\Omega_{p}=0
$$

where $\omega$ is the wave frequency in the $\mathrm{SW}$ rest frame, $\Omega_{p}$ is the proton gyrofrequency, $\mathrm{k}_{\|}$ is the component of the wave vector parallel to the background magnetic field, and $\mathrm{V}_{r}$ is the parallel component of the resonant ion velocity (in the SW frame). The observed wave frequency $\left(\omega_{s c}=2 \pi f_{S C}\right)$ is Doppler shifted as a result of the relative motion be- 

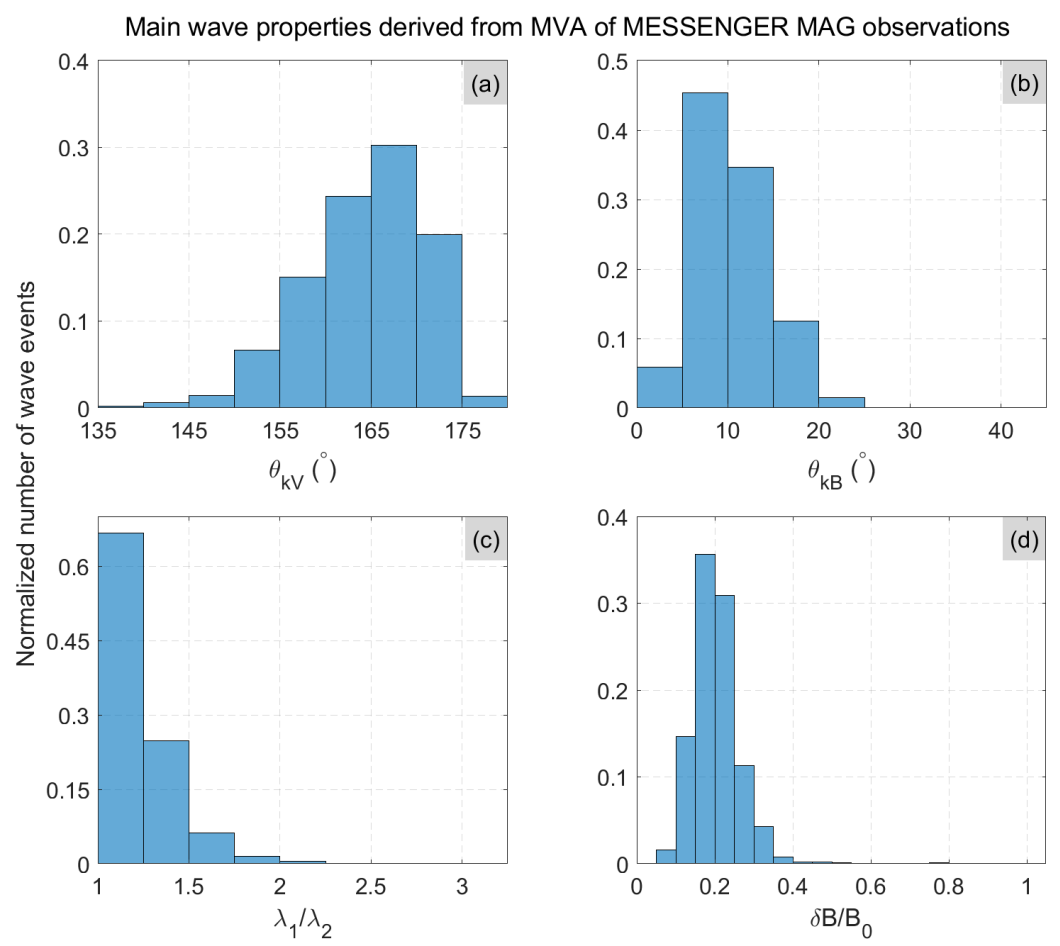

Figure 2. Normalized number of identified waves events rate as a function of $\theta_{k V}$ (Panel a), $\theta_{k B}$ (Panel b), $\lambda_{1} / \lambda_{2}$ (Panel c) and the normalized wave amplitude (Panel d).

tween the spacecraft and SW reference frame. Thus, the observed wave frequency is $\omega_{s c}$ $=\omega+\mathbf{k} \cdot \mathbf{V}_{\mathbf{s w}}$. Making use of Equation (1) we obtain:

$$
\omega_{s c}=\omega+\left(\omega+\Omega_{p}\right) \frac{V_{s w}}{V_{r}} \frac{\cos \left(\theta_{k V}\right)}{\cos \left(\theta_{k B}\right)}
$$

The value of $a=\omega / \Omega_{p}$ near the wavenumber of maximum growth of the ion-ion right hand instability depends on several plasma parameters, e.g., the beam density and drift velocity. However, at least for beam densities between 0.01 and 0.1 the total electron density and fast beams (with respect to the Alfvén speed), $a$ does not depend on $|B|$ (Gary, 1993). Making use of this condition in Equation (2), we can expect an increasing trend between $\left|w_{s c}\right|$ and the background magnetic field (Hoppe and Russell, 1982), if the factor $\frac{V_{s w}}{V_{r}} \frac{\cos \left(\theta_{k V}\right)}{\cos \left(\theta_{k B}\right)}$ does not depend strongly on $|B|$.

Figure (3a) shows the observed wave frequency of all identified events as a function of the corresponding IMF magnitude. We find an increasing trend between the observed $\left|f_{S C}\right|$ and $|B|$, with $\left|f_{S C}\right|$ and $|B|$ ranging between $0.068 \mathrm{~Hz}$ and $0.366 \mathrm{~Hz}$, and 
3.2 Implications for the Speed of Backstreaming Protons in the Foreshock of Mercury

Given Equation (2), we estimate the ratio between the particle velocity parallel to the magnetic field (in the $\mathrm{SW}$ reference frame) and the SW speed, $V_{r} / V_{s w}$, as follows:

$$
\frac{V_{r}}{V_{S W}}=\frac{(1+a) \cos \left(\theta_{k V}\right)}{\left[\left(\omega_{s c} / \Omega_{p}\right)-a\right] \cos \left(\theta_{k B}\right)}
$$

We consider $a \sim 0.15$, a value close to what was reported for ULF waves at the Earth's foreshock (e.g., Mazelle et al., 2003), and also consistent with Gary (1978). Figure (4a) shows the normalized histogram of $V_{r} / V_{s w}$ for all the analyzed events. We find that $\left\langle V_{r} / V_{s w}>+\sigma\left(V_{r} / V_{s w}\right)=1.66 \pm 0.25\right.$, with $V_{r} / V_{s w}$ ranging between 0.95 and 2.6, range that is very close to what was predicted for Mercury (1.2-2.2) (Hoppe and Russell, 1982). Reported values of $V_{r} / V_{s w}$ for Venus $(1.7,1.9)$, Earth $(2.5 \pm 0.3)$, and Jupiter $(2.1,2.3)$ are on the same order to what we find for Mercury. These results show that the observed wave frequencies in these planetary foreshocks are consistent with resonance with beams of protons of similar energy, with speeds ranging between $\sim 1$ and $\sim 2.5$ the SW speed.

For easy comparison with several papers, Figure (4b) shows the histogram for $P_{g c}=$ $V_{g c} / V_{s w}$, that is, the ratio between the guiding center velocity of a backstreaming particle in the foreshock region (in the spacecraft reference frame) and the SW speed. Meziane and D'Uston (1998) showed that:

$$
P_{g c}=\sqrt{1+\left(V_{r} / V_{s w}\right)^{2}-2\left(V_{r} / V_{s w}\right) \cos \left(\theta_{B X}\right)}
$$

where $\theta_{B X}$ is the angle that the $\mathrm{X}$-axis makes with the IMF direction. We find that the waves identified in the Hermean foreshock have $\left\langle P_{g c}>+\sigma\left(P_{g c}\right)=0.76 \pm 0.27\right.$, as a result of the relatively low IMF cone angle range observed around Mercury (e.g., James et al., 2017). Studies on other planetary foreshocks reported larger values for $\left\langle P_{g c}\right\rangle$ (e.g., Shan et al., 2018, and references therein). Indeed, Shan et al. (2018) and Andrés et al. (2015) reported that $P_{g c}=1.07$ (for $\theta_{B X}=36^{\circ}$ ) and $P_{g c}=1.05 \pm 0.01$ (for $\left.\theta_{B X}=45^{\circ}\right)$, when restricted to the ULF wave boundary in the Venusian and Earth's foreshock, respectively. The finding by Andrés et al. (2015) is in approximate agreement with results $\left(P_{g c}=1.11 \pm 0.04\right)$ reported in Meziane and D'Uston (1998), and contrasts with the value associated for the field aligned beam-gyrating boundary $\left(P_{g c}=1.68 \pm\right.$ 0.08), derived in Meziane et al. (2004). This difference might be explained if the latter 

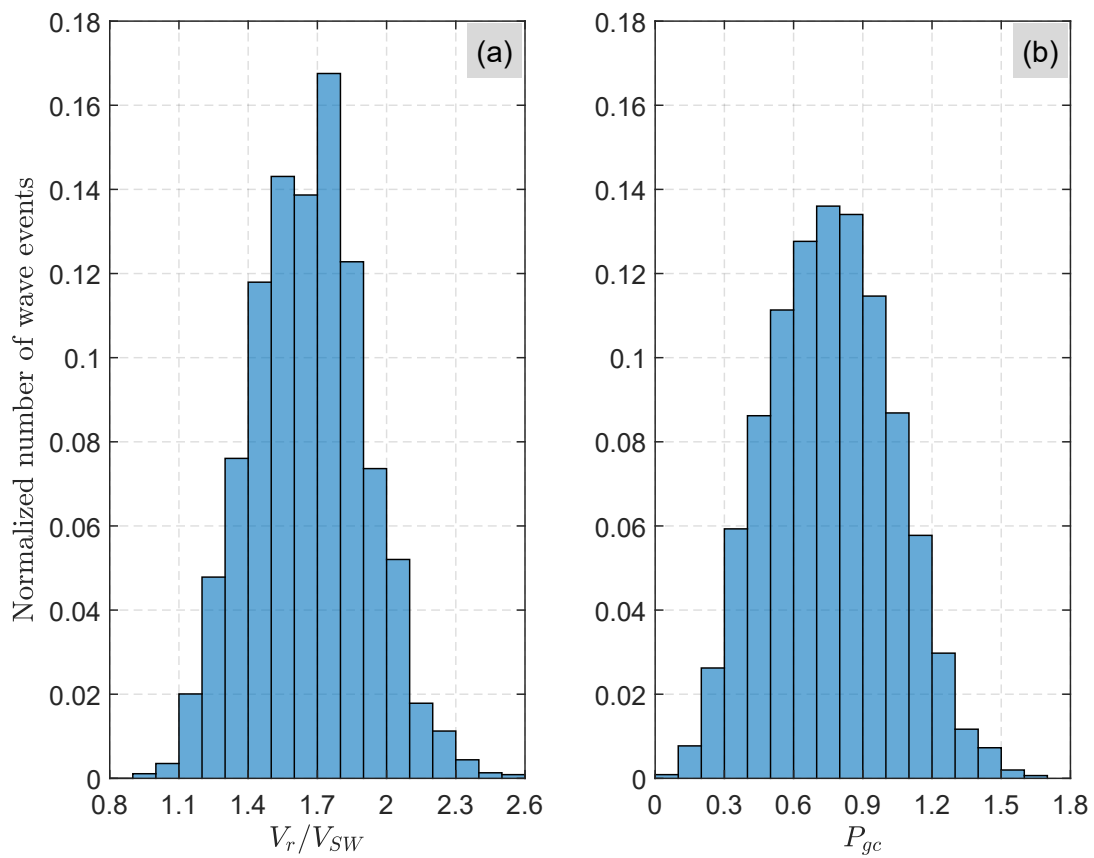

Figure 4. Normalized number of wave events as a function of $V_{r} / V_{s w}$ (Panel a) and $P_{g c}$ (Panel b).

boundary is the same (or close) to the quasi-monochromatic ULF wave boundary. A comparison between the reported $P_{g c}$ values and the associated $\theta_{B X}$ for these planetary foreshocks and the Hermean foreshock supports the idea that the $V_{r} / V_{s w}$ range is similar for these magnetospheric environments. It is also worth noticing that the mean values and standard deviation of $V_{r} / V_{s w}$ and $P_{g c}$ do not vary strongly when $a$ ranges between 0.05 and 0.15 , in association with changes in plasma properties affecting the maximum linear wave growth rate of the ion-ion right hand instability (Gary, 1993). Indeed, $\left\langle V_{r} / V_{s w}>\right.$ $+\sigma\left(V_{r} / V_{s w}\right)=1.79 \pm 0.31$ and $\left\langle P_{g c}>+\sigma\left(P_{g c}\right)=0.88 \pm 0.33\right.$ for $a=0.05$ and $<V_{r} / V_{s w}>+\sigma\left(V_{r} / V_{s w}\right)=1.72 \pm 0.28$ and $\left\langle P_{g c}>+\sigma\left(P_{g c}\right)=0.82 \pm 0.30\right.$ for $a=0.10$.

To our knowledge, the only study that has provided sufficient information to derive $P_{g c}$ for the Hermean foreshock is Jarvinen et al. (2019). These authors performed a hybrid simulation of the interaction of Mercury with the SW under conditions proper of perihelion. If we assume that the field aligned beam that might give rise to the simulated waves of interest has approximately the same energy as the foreshock ions that coexist with the simulated quasi-monochromatic waves (see description of Figure 6), we 
conclude that this simulation suggests that $V_{r} / V_{s w} \sim 1.27$ and $P_{g c} \sim 0.43$. Both estimations are within the computed ranges shown in Figure (4a) and (4b). Note that while this simulation considers a specific set of conditions, Figure 4 is associated with wave events observed along all Mercury's eccentric orbit around the Sun. Indeed, the energy gained by the backstreaming protons is partly controlled by the size of the bow shock and the tangential convective electric field, among other factors that vary with the heliocentric distance (e.g., Meziane et al., 2017). A detailed analysis on the possible acceleration mechanisms of the backstreaming protons in the Hermean foreshock is beyond the scope of this article.

Moreover, if nonlinear wave-particle trapping takes place in the Hermean foreshock, we could expect to observed gyrophase bunched distribution functions. By applying the theoretical framework considered in (Mazelle et al., 2000), (Mazelle et al., 2003) and Romanelli, Mazelle \& Meziane (2018), we find that quasi-monochromatic waves with $\delta B /\left|\mathbf{B}_{\mathbf{0}}\right| \sim$ 0.2 that might arise by field-aligned beams will tend to trap particles with the same energy (in the wave rest frame) around a pitch angle of $\sim 40^{\circ}$. A future analysis of velocity distribution functions provided by MESSENGER Fast Imaging Plasma Spectrometer and by the upcoming Bepi-Colombo mission (Benkhoff et al., 2010) should be performed to test this prediction.

Finally, we compute the ratio between the number of time intervals with waves and the number of intervals when MESSENGER is connected to the shock. We determine that the occurrence rate of the lowest frequency waves is approximately $0.5 \%$. This number varies depending upon the wave selection criteria. However, if we consider a less restrictive criteria based only on PSD properties (e.g., $r=2$ ), this ratio is $\sim 1.5 \%$, still very low. This low occurrence rate value is in agreement with initial observations by Le et al. (2013) and could be due to several factors: relatively low backstreaming ion fluxes due to the low SW Alfvénic Mach numbers around Mercury; the small size of the Hermean foreshock where the waves can grow once the instability occurs; and/or the short timescales over which the external conditions may vary, that could disturb the growing phase of the waves. As reported in Le et al. (2013), MESSENGER has not detected these waves in the steepening waveform, often observed in the terrestrial foreshock. The lack or potentially lower wave occurrence rate in such compressive stage is consistent with the small wave amplitude and propagation angles shown in Figure 2 and the lack of steep- 
ened waveforms and shocklets in the upstream region of low Mach number interplanetary shocks (Blanco-Cano et al., 2013; Blanco-Cano et al., 2016).

\section{Conclusions}

We performed the first statistical analysis of the main properties of ultra-low frequency waves in Mercury's foreshock, making use of high-time resolution MESSENGER magnetic field measurements. We find that waves with a power spectral density peak in the $0.05-0.41 \mathrm{~Hz}$ range are close to be circularly polarized, they propagate quasi-parallel to the background magnetic field $\left(\sim 10^{\circ}\right)$, quasi antiparallel to the solar wind velocity $\left(\sim 165^{\circ}\right)$ and have relatively low normalized wave amplitude $\left(\delta B /\left|\mathbf{B}_{\mathbf{0}}\right| \sim 0.2\right)$.

These waves have similar properties to the '30 second waves' observed in the Earth's foreshock, previously associated with fast magnetosonic waves generated by backstreaming protons. In sharp contrast with the terrestrial foreshock, the normalized wave amplitude and the occurrence rate of these waves $(\sim 0.5 \%)$ seems relatively low in the Hermean foreshock, suggesting significant lower backstreaming protons fluxes likely due to the relatively low solar wind Alfvénic Mach number. These differences could also be related to the smaller foreshock size and/or more variable solar wind conditions. An analysis of MESSENGER MAG observations focused on the conditions that favor the presence of these waves will be performed in a future study to elucidate what is the main constraining factor.

Finally, we estimate that the velocity of resonant backstreaming protons parallel to the magnetic field in the solar wind reference frame (normalized with the solar wind speed) ranges between $0.95-2.6$. These results are consistent with particles being accelerated at the Hermean bow shock up to energies on the same order of other solar system planetary bow shocks, even under the low solar wind Alfvénic Mach regime around Mercury. As reported in Hoppe and Russell (1982), the apparent generality of this phenomena in the solar system suggests that similar acceleration mechanisms might take place in the bow shocks of exoplanets, and might provide a source of cosmic rays.

\section{Acknowledgments}

N.R. is an Assistant Research Scientist at NASA Goddard Space Flight Center, and University of Maryland Baltimore County hired through a cooperative agreement with Cen- 
ter for Research and Exploration in Space Sciences \& Technology II. DJG and GAD were supported by the NASA ROSES Discovery Data Analysis program under grant NNX16AJ05G. MESSENGER Magnetometer data used in this study are publicly available through the Planetary Data System (https://pds-ppi.igpp.ucla.edu/index.jsp).

\section{References}

Anderson, B. J., Acuna, M. H., Lohr, D. A., Scheifele, J., Raval, A., Korth, H., Slavin, J. A. (2007). The Magnetometer instrument on MESSENGER. Space Science Reviews, 131(1-4), 417-450. https://doi.org/10.1007/s11214-007-9246-7

Anderson, B. J., Johnson, C. L., Korth, H., et al. 2011, Sci, 333, 1859.

Andrés, N., K. Meziane, C. Mazelle, C. Bertucci and D. Gómez (2015), The ULF wave foreshock boundary: Cluster observations, J. Geophys. Res. Space Physics, 120, 4181-4193, doi:10.1002/2014JA020783.

Asbridge, J. R., S. J. Bame, and I. B. Strong (1968), Outward flow of protons from the Earth's bow shock, J. Geophys. Res., 73, 5777.

Benkhoff, J., J. van Casteren, H. Hayakawa, M. Fujimoto, H. Laakso, M. Novara, P. Ferri, H.R. Middleton, and R. Ziethe (2010), BepiColombo-Comprehensive exploration of Mercury: Mission overview and science goals, Planet. Space Sci., doi:10.1016/j.pss.2009.09.020.

Blanco-Cano, X., P. Kajdic, E. Aguilar-Rodriguez, C. T. Russell, L. K. Jian, and J. G. Luhmann (2013), STEREO observations of interplanetary shocks and foreshocks, Solar Wind 13: Proceedings of the Thirteenth International Solar Wind Conference, AIP Conf. Proc. 1539, edited by N. V. Pogorelov and G. P. Zank, American Institute of Physics, New York, doi.org/10.1063/1.4811005. Blanco-Cano, X., P. Kajdic, E. Aguilar- Rodríguez, C. T. Russell, L. K. Jian, and J. G. Luhmann (2016), Interplanetary shocks and foreshocks observed by STEREO during 2007-2010, J. Geophys. Res. Space Physics, 121, 992-1008, doi:10.1002/2015JA021645.

Bonifazi, C., and G. Moreno (1981), Reflected and diffuse ions backstreaming from the Earth's bow shock. 1: Basic properties, J. Geophys. Res., 86, 4397-4413.

Brinca, A. (1991). Cometary linear instabilities: From profusion to perspective, Cometary plasma processes geophysical monograph (Vol. 61, pp. 211-221). Washington, DC: American Geophysical Union. 
Biskamp, D. (1973), Collisionless shock waves in plasmas, Nucl. Fusion, 13, 719.

Burgess, D., E. Mobius, and M. Scholer (2012), Ion acceleration at the Earth's bow shock, Space Sci. Rev., 173, 5-47, doi:10.1007/s11214-012- 9901-5.

Eastwood, J. P., Lucek, E. A., Mazelle, C., Meziane, K., Narita, Y., Pickett, J., \& Treumann, R. A. (2005). The foreshock. Space Science Reviews, 118, 41-94.

Fairfield, D. H. (1974), Whistler waves observed upstream from collisionless shocks, J. Geophys. Res., 79(10), 1368-1378, doi:10.1029/JA079i010p01368.

Fairfield, D. H., and K. W. Behannon (1976), Bow Shock and magnetosheath waves at Mercury, J. Geophys. Res., 81(22), 3897-3906, doi:10.1029/JA081i022p03897.

Gary S. P., 1978, Nuclear Fusion, 18, 327.

Gary, S. P., Akimoto, K., and Winske, D. (1989), Computer simulations of cometary-ion/ion instabilities and wave growth, J. Geophys. Res., 94(A4), 3513- 3525, doi:10.1029/JA094iA04p03513.

Gary, S.P., 1991. Electromagnetic ion/ion instabilities and their consequences in space plasmas: a review. Space Science Reviews 56, 373-415.

Gary, S.P., 1993. Theory of space plasma microinstabilities. In: Cambridge Atmospheric and Space Series. Cambridge University Press, Cambridge.

Gershman, D. J., J. A. Slavin, J. M. Raines, T. H. Zurbuchen, B. J. Anderson, H. Korth, D. N. Baker, and S. C. Solomon (2013), Magnetic flux pileup and plasma depletion in Mercury's subsolar magnetosheath, J. Geophys. Res. Space Physics, 118, 7181-7199, doi:10.1002/2013JA019244.

Greenstadt, E., and L. Baum (1986), Earth's compressional foreshock boundary revisited: Observations by the ISEE 1 magnetometer, J. Geophys. Res., 91(A08), 9001-9006.

Greenstadt, E. W., I. M. Green, G. T. Inouye, A. J. Hundhausen, S. J. Bame, and I. B. Strong (1968), Correlated magnetic field and plasma observations of the Earth's bow shock, J. Geophys. Res., 73(1), 51-60, doi:10.1029/ JA073i001p00051.

Hoppe, M. . M., and C. T. Russell (1982), Particle acceleration at planetary bow shock waves, Nature, 295, 41.

Jarvinen, J., M Alho, E Kallio, T I Pulkkinen, Ultra-low frequency waves in the ion foreshock of Mercury: A global hybrid modeling study, Monthly Notices of the 
Royal Astronomical Society, stz3257, https://doi.org/10.1093/mnras/stz3257.

James, M. K., S. M. Imber, E. J. Bunce, T. K. Yeoman, M. Lockwood, M. J. Owens, and J. A. Slavin (2017), Interplanetary magnetic field properties and variability near Mercury's orbit, J. Geophys. Res. Space Physics, 122, 7907-7924, doi:10.1002/2017JA024435.

Kennel, C. F., J. P. Edmiston, and T. Hada (1985), A quarter century of collisionless shock research, in Collisionless Shocks in the Heliosphere: A Tutorial Review, Geophys. Monogr. Ser., vol.34, edited by B. T. Tsurutani, and R. G. Stone, p. 1, AGU, Washington, DC.

Le, G., P. J. Chi, X. Blanco-Cano, S. Boardsen, J. A. Slavin, and B. J. Anderson (2013), Upstream ultra-low frequency waves in Mercury's foreshock region: MESSENGER magnetic field observations, J. Geophys. Res. Space Physics, 118, 2809-2823, doi:10.1002/jgra.50342.

Masters, A., J. A. Slavin, G. A. DiBraccio, T. Sundberg, R. M. Winslow, C. L. Johnson, B. J. Anderson, and H. Korth (2013), A comparison of magnetic overshoots at the bow shocks of Mercury and Saturn, J. Geophys. Res. Space Physics, 118, 4381-4390, doi:10.1002/jgra.50428.

Mazelle, C., Le Queau, D., \& Meziane, K. (2000). Nonlinear wave-particle interaction upstream from the Earth's bow shock. Nonlinear Processes in Geophysics, $77,185-190$.

Mazelle, C., Meziane, K., LeQueau, D., Wilber, M., Eastwood, J. P., Reme, H.,...,Balogh, A. (2003). Production of gyrating ions from nonlinear waveparticle interaction upstream from the Earth's bow shock: A case study from Cluster-CIS. Planetary and Space Science, 51, 785-795.

Meziane, K., and C. D'Uston (1998), A statistical study of the upstream intermediate ion boundary in the Earth's foreshock, Ann. Geophys., 16, 125-133.

Meziane, K., et al. (2004), Simultaneous observations of field-aligned beams and gyrating ions in the Terrestrial foreshock, J. Geophys. Res., 109, A05107, doi:10.1029/2003JA010374.

Meziane, K., C. X. Mazelle, N. Romanelli, D. L. Mitchell, J. R. Espley, J. E. P. Connerney, A. M. Hamza, J. Halekas, J. P. McFadden, and B. M. Jakosky (2017), Martian electron foreshock from MAVEN observations, J. Geophys. Res. Space Physics, 122, 1531-1541, doi:10.1002/2016JA023282. 
Paschmann, G., N. Sckopke, J. R. Asbridge, S. J. Bame, and J. T. Gosling (1980), Energization of solar wind ions by reflection from the Earth's bow shock, J. Geophys. Res., 85(A9), 4689-4693, doi:10.1029/ JA085iA09p04689.

Romanelli, N., et al. (2016), Proton cyclotron waves occurrence rate upstream from Mars observed by MAVEN: Associated variability of the Martian upper atmosphere, J. Geophys. Res. Space Physics, 121, 11,113-11,128, doi:10.1002/2016JA023270.

Romanelli, N., Mazelle, C., \& Meziane, K. (2018). Nonlinear wave-particle interaction: Implications for newborn planetary and backstreaming proton velocity distribution functions. Journal of Geophysical Research: Space Physics, 123, 1100-1117. https://doi.org/10.1002/2017JA024691

Russell, C. T., M. M. Hoppe, and W. A. Livesey (1982), Overshoots in planetary bow shocks, Nature, 296, 45-48.

Russell, C. T. (1985), Planetary bow shocks, in Collisionless Shocks in the Heliosphere: Reviews of Current Research, Geophys. Monogr. Ser., vol. 35, edited by R. G. Stone and B. T. Tsurutani, pp. 109 - 130, AGU, Washington, D. C. Shan, L., Mazelle, C., Meziane, K., Romanelli, N., Ge, Y. S., Du, A., ... Zhang, T. (2018). The quasimonochromatic ULF wave boundary in the Venusian foreshock: Venus Express observations. Journal of Geophysical Research: Space Physics, 123, 374-384. https://doi.org/10.1002/2017JA024054

Slavin, J. A., and R. E. Holzer (1981), Solar wind flow about the terrestrial planets 1. Modeling bow shock position and shape, J. Geophys. Res., 86(A13), 11,401-11,418, doi:10.1029/JA086iA13p11401.

Slavin, J. A., Middleton, H. R., Raines, J. M., Jia, X., Zhong, J., Sun, W.-J., et al (2019). MESSENGER observations of disappearing dayside magnetosphere events at Mercury. Journal of Geophysical Research: Space Physics, 124, 6613-6635. https://doi.org/ 10.1029/2019JA026892.

Solomon, S. C., McNutt, R. L. Jr., Gold, R. E., \& Domingue, D. L. (2007). MESSENGER mission overview. Space Science Reviews, 131 (1-4), 3-39. https://doi.org/10.1007/s11214-007-9247-6

Sonnerup, B. U. O. (1969), Acceleration of particles reflected at a shock front, J. Geophys. Res., 74(5), 1301-1304, doi:10.1029/JA074i005p01301.

Sonnerup, B. U. O., and M. Scheible (1998), Minimum and maximum variance anal- 
ysis, in Analysis Methods for Multi-Spacecraft Data, ISSI Scientific Reports Series, vol. 1, pp. 185-220, edited by G. Paschmann and P. Daly, ESA Publications Division, Noordwijk, Netherlands.

Song, P. and C. T. Russell: 1999, 'Time series data analyses in space plasmas'. Space Sci. Rev. 87, 387-463.

Wilson, L. B. (2016). Low frequency waves at and upstream of collisionless shocks. In Low-frequency waves in space plasmas, Geophysical Monograph Series (Vol. 216, pp. 269-291). Hoboken, NJ: John Wiley. https://doi.org/10.1002/9781119055006.ch16

Winslow, R. M., Anderson, B. J., Johnson, C. L., Slavin, J. A., Korth, H., Purucker, M. E., et al. (2013). Mercury's magnetopause and bow shock from MESSENGER observations. Journal of Geophysical Research: Space Physics, 118, 2213-2227. https://doi.org/10.1002/ jgra.50237. 
Main wave properties derived from MVA of MESSENGER MAG observations
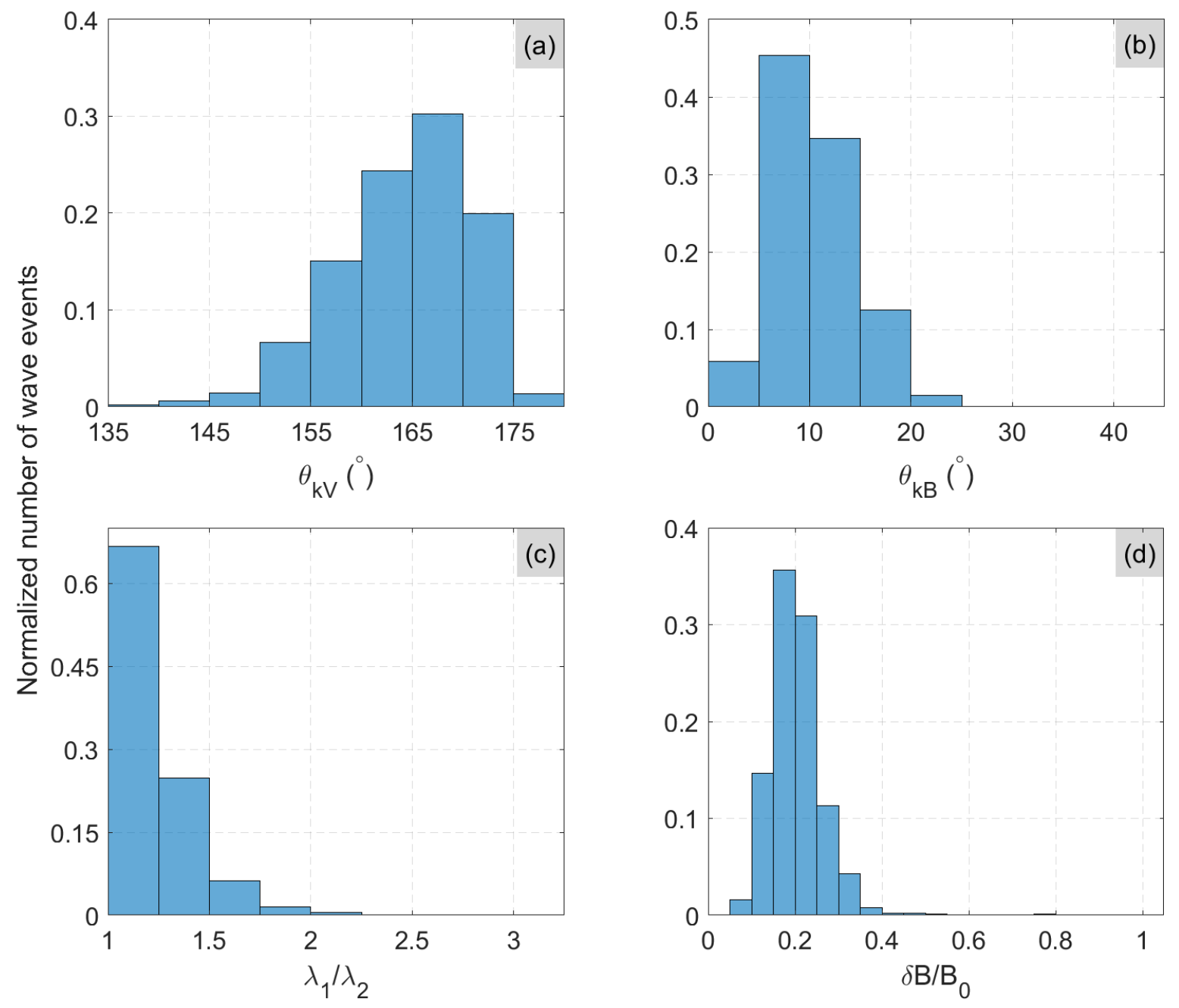


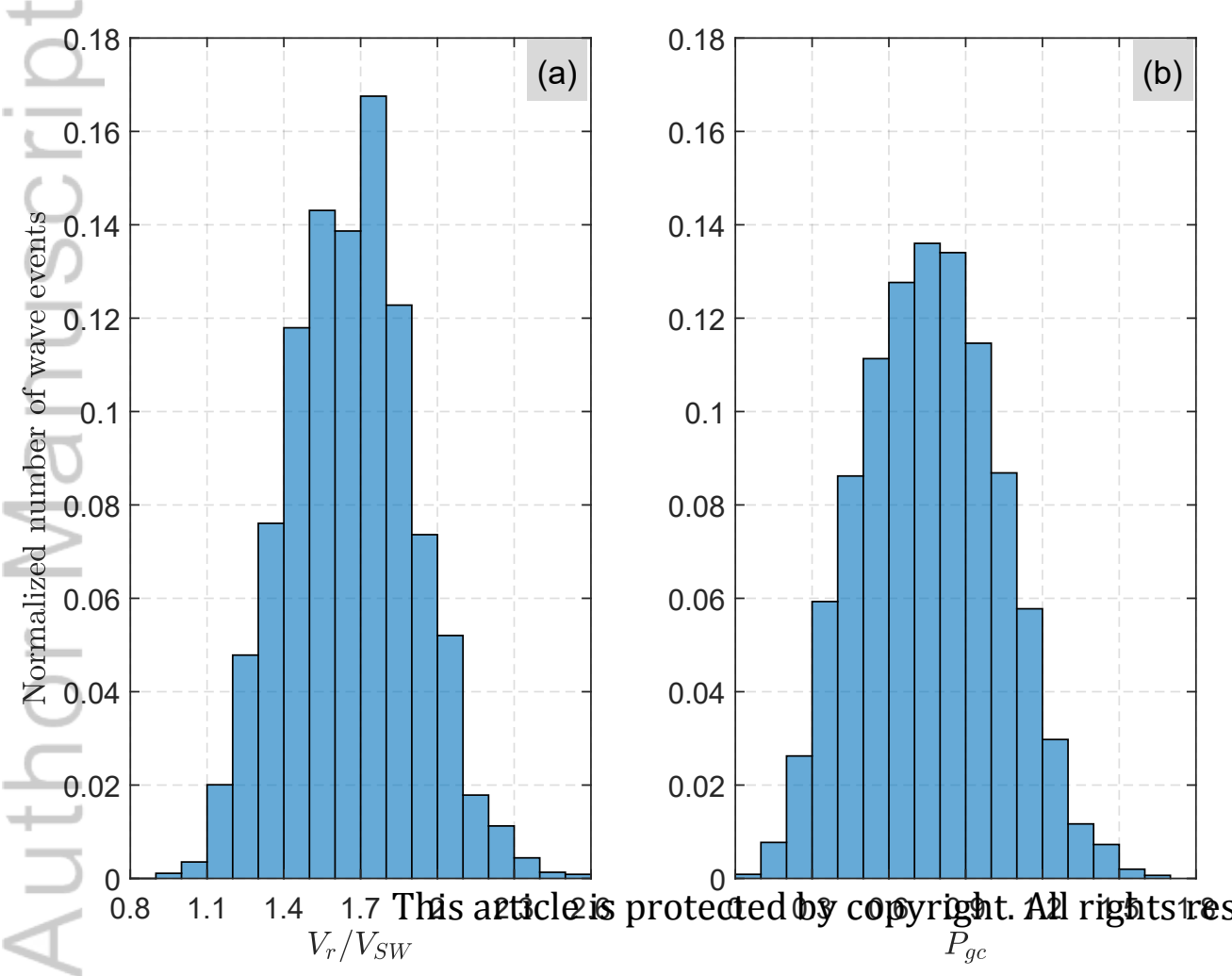

